\title{
Comment on: "The Role of Chronic Disease, Obesity, and Improved Treatment and Detection in Accounting for the Rise in Healthcare Spending Between 1987 and 2011"
}

\author{
Afschin Gandjour ${ }^{1}$
}

Published online: 25 February 2016

(c) Springer International Publishing Switzerland 2016

The article by Kenneth Thorpe and colleagues published recently in Applied Health Economics and Health Policy aims at identifying drivers of health expenditure growth in the USA for the period from 1987 to 2011 [1]. Specifically, the paper analyzes the contribution of selected chronic conditions including obesity. To this end, it decomposes total spending growth and spending growth for each chronic condition into three components: (1) change in treated prevalence, (2) change in treatment costs per individual, and (3) change in population size. These three factors explain $100 \%$ of spending growth. The authors find that the $30.6 \%$ of total spending growth is attributable to the increase in the prevalence of chronic conditions. As obesity accounts for $6.4 \%$ of this growth, it is responsible for $2.0 \%(30.6 \times 6.4 \%)$ of total spending growth. The authors conclude that "policies aimed at reducing obesity in the USA might yield important savings in the future".

The decomposition approach used by the paper hinges on the assumption that the three drivers of spending growth are independent. That is, population growth is exogenous to chronic conditions such as obesity. Hence, a change in treated prevalence does not affect population size. However, this assumption is frequently violated: most chronic conditions affect not only morbidity but also mortality. Obesity for example, does not only affect expenditure growth by a change in prevalence but also by its effect on population growth. That is, by reducing length of life, obesity contributes to a decrease in population growth and hence slows down expenditure growth. In fact, there have

Afschin Gandjour

a.gandjour@fs.de

1 Frankfurt School of Finance and Management, Sonnemannstr. 9-11, 60314 Frankfurt, Germany been concerns that average life expectancy in the USA might decrease due to obesity [2]. Furthermore, there is evidence from the Netherlands [3] and more recently from the USA [4], that obese persons have lower lifetime costs compared with those of normal weight due to a shorter life expectancy. Based on this evidence the financial impact of obesity through its reduction in population growth would be larger than through its increase in the prevalence of chronic conditions. While evidence on this question remains mixed [5], the important point is that population growth is not exogenous but negatively associated with disease prevalence. Policy recommendations need to account for this relationship.

Acknowledgments This letter received no specific grant from any funding agency in the public, commercial or not-for-profit sectors. I declare that I have no competing interests.

\section{References}

1. Thorpe KE, Allen L, Joski P. The role of chronic disease, obesity, and improved treatment and detection in accounting for the rise in healthcare spending between 1987 and 2011. Appl Health Econ Health Policy. 2015;13(4):381-7.

2. Olshansky SJ, Passaro DJ, Hershow RC, Layden J, Carnes BA, Brody J, Hayflick L, Butler RN, Allison DB, Ludwig DS. A potential decline in life expectancy in the United States in the 21st century. N Engl J Med. 2005;352(11):1138-45.

3. van Baal PH, Polder JJ, de Wit GA, Hoogenveen RT, Feenstra TL, Boshuizen HC, Engelfriet PM, Brouwer WB. Lifetime medical costs of obesity: prevention no cure for increasing health expenditure. PLoS Med. 2008;5(2):e29.

4. Gaudette É, Goldman DP, Messali A, Sood N. Do statins reduce the health and health care costs of obesity? Pharmacoeconomics. 2015;33(7):723-34.

5. Gandjour A. Cost-effectiveness of preventing weight gain and obesity: what we know and what we need to know. Expert Rev Pharmacoecon Outcomes Res. 2012;12(3):297-305. 